\title{
'Epidemic neuromyasthenia' at the Hospital for Sick Children, Great Ormond Street, London
}

\author{
M. J. Dillon \\ M.B., M.R.C.P., D.C.H. \\ The Hospital for Sick Children, Great Ormond Street, London
}

\begin{abstract}
Summary
An outbreak of an illness with features in common with 'epidemic neuromyasthenia' affected the staff of the Hospital for Sick Children in London between August 1970 and January 1971. At least 145 cases were observed and the majority of these were nurses. Symptomatology was protean, clinical findings minimal and relapses frequent. Care was taken to minimize anxiety and fear in a vulnerable population, and laboratory investigations were purposefully limited in number for this reason. In general, laboratory findings, including virological investigations were negative. A high incidence of anti-complementary activity and the presence of ill defined aggregates in some acute sera on electron microscopy were interesting and possibly significant findings suggesting the presence of immune complexes. These findings, plus the ability of lymphocytes from some patients to proliferate in vitro, were thought to represent possible evidence of an infective process. Although no children were affected during the 1970 outbreak, it is interesting that seven children have recently been referred to the hospital with features compatible with 'epidemic neuromyasthenia'.
\end{abstract}

\section{Introduction}

An illness with features in common with what has become known as 'epidemic neuromyasthenia' (ENM) (Acheson, 1959; Henderson and Shelokov, 1959; Shelokov, 1972; Parish, 1974) affected the staff of the Hospital for Sick Children, Great Ormond Street, London, between August 1970 and January 1971. This outbreak has already been reported in detail (Dillon et al., 1974) and, therefore, only a brief account of it will be given in this paper plus some recent data on the sporadic occurrence of this condition in childhood.

\section{Patients and Methods}

\section{Epidemiology}

At least 145 cases were observed during the 1970-1971 outbreak at the Hospital for Sick
Children, and the majority of these were nurses. Between August 1970 and January 1971136 cases were seen followed by a few sporadic cases between February and June 1971. Of those affected, 124 were nurses (103 student nurses and twenty-one senior nurses), six were doctors and there were fifteen cases amongst other hospital staff. Most of the patients were women but at least four men were affected. Of those, two were doctors and two were on the administrative staff. The staff numbered about 1900 and therefore the minimum incidence of symptomatic disease was $7-8 \%$. It was interesting that none of the children who were in-patients during this period had similar symptoms. Of the 1900 staff, 800 were nurses, 190 were doctors and the remainder were other personnel. Thus $15 \%$ of the nurses were affected, $3 \%$ of the medical staff and $2 \%$ of the remainder. There were two fairly distinct waves, the first between mid-August and the end of September, 1970, and the second which finished towards the end of January 1971 (Fig. 1). There were thirty-three cases in the first wave, most of whom were student nurses and over $50 \%$ of these were attending daily classes in the Nurses' training school immediately before the start of the outbreak. A sister tutor who taught these nurses was also affected. During the second wave, all levels of nursing staff were affected together with some doctors, domestic staff and others.

\section{Clinical manifestations}

As far as clinical manifestations were concerned, it was found that symptomatology was protean and physical findings minimal. Table 1 lists the symptoms and their incidence seen in those affected. One symptom which does not appear in the table because it was difficult to quantify, was rapid fatigability reported on exercise. Table 2 lists the abnormal physical signs found in the 145 patients.

The initial features of the illness lasted 2-3 weeks and then gradually resolved during the next 2-3 months. However, a striking feature was the occurrence of symptomatic relapses over a prolonged 


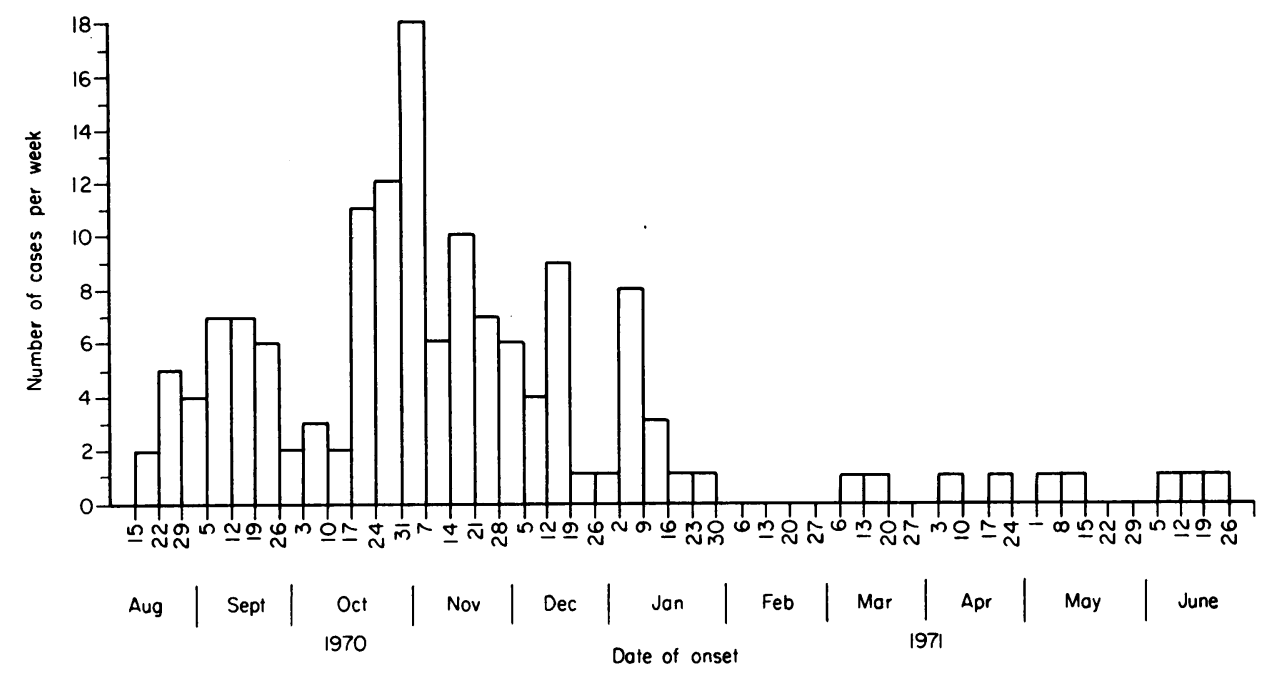

Fig. 1. Occurrence of cases between August 1970 and June 1971. (From Dillon et al., 1974, by permission of the editor of British Medical Journal.)

TABLE 1. Symptoms and their incidence in 145 patients. (From Dillon et al., 1974, by permission of the editor of the British Medical Journal.)

\begin{tabular}{llll} 
& $\begin{array}{c}\text { No. }(\%) \\
\text { of } \\
\text { patients }\end{array}$ & & $\begin{array}{c}\text { No. (\%) } \\
\text { of } \\
\text { patients }\end{array}$ \\
\hline Headache & $82(56 \cdot 5)$ & Abdominal pain & $8(5 \cdot 5)$ \\
Sore throat & $60(41 \cdot 3)$ & Photophobia & $8(5 \cdot 5)$ \\
Nausea & $53(36 \cdot 5)$ & Diarrhoea & $8(5 \cdot 5)$ \\
Back pain & $39(26 \cdot 8)$ & Earache & $7(4 \cdot 8)$ \\
Malaise & $38(26 \cdot 2)$ & Loss of voice (laryngitis) & $6(4 \cdot 1)$ \\
Vomiting & $38(26 \cdot 2)$ & Sensory symptoms (paraesthesiae) & $5(3 \cdot 4)$ \\
Neck pain & $37(25 \cdot 5)$ & Faintness & $4(2 \cdot 7)$ \\
Tiredness & $28(19 \cdot 3)$ & Jaw pain & $3(2 \cdot 0)$ \\
Limb pains & $25(17 \cdot 2)$ & Bladder symptoms & $3(2 \cdot 0)$ \\
Depression & $18(12 \cdot 4)$ & Anorexia & $3(2 \cdot 0)$ \\
Dizziness/giddiness & $15(10 \cdot 3)$ & Subjective limb weakness & $2(1 \cdot 3)$ \\
Sore eyes & $14(9 \cdot 6)$ & Blurred vision & $2(1 \cdot 3)$ \\
Cough & $14(9 \cdot 6)$ & Diplopia & $1(0 \cdot 6)$ \\
Coryza & $10(6 \cdot 8)$ & Painful joints & $1(0 \cdot 6)$ \\
Chest pain & $9(6 \cdot 2)$ & & \\
\hline
\end{tabular}

period in at least twenty-eight patients. Intervals between apparent recovery and relapse varied from 2-6 months, but even during the period of recovery the patients were not restored to normal health. The total duration of the illness was difficult to define, some patients were free of symptoms after 12 months but in others symptomatic relapses occurred for several years after the initial illness.

\section{Treatment}

Treatment was unsatisfactory and consisted of symptomatic measures. Simple analgesics were of little value but pentazocine, dihydrocodeine and dextropropoxyphene were more helpful for the headaches and back pain. Anti-emetics were usefur but antidepressants and short-wave diathermy weres of little value.

\section{Laboratory investigations}

In the first fortnight of the outbreak a deliberate? decision was taken to limit the number of investiga- 0 tions, especially invasive procedures, in order to 
TABLE 2. Abnormal physical signs and their incidence in 145 patients. (From Dillon et al., 1974, by permission of the editor of the British Medical Journal.)

\begin{tabular}{lc}
\hline & $\begin{array}{c}\text { No. }(\%) \\
\text { of patients }\end{array}$ \\
\hline Pharyngeal injection & $88(60 \cdot 8)$ \\
Cervical lymph node enlargement & $34(23 \cdot 4)$ \\
Neck stiffness & $16(11 \cdot 0)$ \\
Injected conjunctivae & $14(9 \cdot 6)$ \\
Pyrexia & $13(8 \cdot 9)$ \\
Photophobia & $8(5 \cdot 5)$ \\
Rash & $6(4 \cdot 1)$ \\
Slight splenomegaly & $2(1 \cdot 3)$ \\
Distended retinal veins & $2(1 \cdot 3)$ \\
Papilloedema & $1(0 \cdot 6)$ \\
Sluggish pupillary reactions to light & 1 \\
Fits in known epileptic & $10 \cdot 6)$ \\
Arthritis & $1(0 \cdot 6)$ \\
\hline
\end{tabular}

prevent anxiety and fear in a theoretically susceptible population. The following investigations were undertaken:

(a) Haematological. Haemoglobin level, total and differential white blood count, erythrocyte sedimentation rates (ESR), and Paul-Bunnell screening test (Monospot, Ortho Diagnostics).

(b) Biochemical. Liver function tests and serum creatine phosphokinase (CPK) levels in some patients.

(\$) Bacteriological. Throat swabs for bacterial pathogens and Mycoplasma pneumoniae. Mycoplasma complement fixing antibody tests were also performed on acute and convalescent sera.

(d) Cerebrospinal fluid examination (CSF). CSF from three patients was examined for cells, protein and sugar levels. Bacteriological and virological examination was also carried out.

(e) Virological investigations.

(i) Attempts to isolate a virus by inoculating nose, throat and rectal swabs into a wide variety of cell cultures (primary and continuous lines of monkey kidney, rabbit kidney, human embryo-lung fibroblasts and human embryo-kidney cells) as well as human embryotrachea cultures (Central Public Health Laboratory, Colindale, London) and suckling mice.

(ii) Serological tests for antibody to the following viruses: cytomegalovirus (CMV), adenovirus, herpes simplex, influenzae A and B, parainfluenzae 1 and 3, mumps and EB virus using complement fixation tests, rubella and measles using haemagglutinationinhibition tests and Coxsackie B virus types 1-6 using virus neutralization tests.

(iii) Electron and immune electron microscopy (Royal Postgraduate Medical School). Serum deposits were examined after differential centrifugation by the electron microscope technique of negative staining. Immune electron microscopy was used to examine acute-phase sera after interaction with recovery-phase sera (Almeida and Waterson, 1969) and similar tests were carried out on supernatant fluids and cell homogenates of lymphocyte cultures from patients in the acute phase of the disease.

(f) Other laboratory investigations. Measurement of immunoglobulins (IgM, IgA and IgG) in acute and convalescent sera; tests for the presence of interferon in acute-phase sera and supernatants of cultured lymphocytes by inhibition of cytopathic effects of Sindbis virus in monolayer cultures of human embryo-lung fibroblasts; phytohaemagglutinin (PHA) stimulation of peripheral lymphocytes and the setting up of long-term cultures of peripheral lymphocytes from three patients in the acute phase of the illness.

\section{Results}

Haematological investigations carried out on fifty-eight patients showed that haemoglobin levels were normal and ESRs not raised. Total white cell counts ranged from $3.0-9.0 \times 10^{9} / 1$ but in five patients the counts ranged from $9 \cdot 5-12.3 \times 10^{9} / 1$ and from $1.6-2.5 \times 10^{9} / 1$ in four. Mean polymorph count was $56 \%$ (range 27-85), and the mean lymphocyte count $36 \%$ (range 12-68) of the total. In twenty of the patients, between $1-5 \%$ of lymphocytes were 'atypical' but in two patients, there were 10 and $70 \%$ atypical cells. The Paul-Bunnell screening tests were positive in forty-eight patients. Liver function tests were normal in eleven patients but there was a slight rise in both transaminases and $\alpha_{2}$ - and $\gamma$-globulin levels in one patient. Serum CPK levels were normal in the small number of patients tested. CSF was normal in the three patients in whom it was examined. Group A $\beta$-haemolytic streptococci were isolated from throat swabs in five patients. M. pneumoniae was not isolated.

\section{Virological investigations}

The only viruses isolated were Coxsackie B1 from the throat swab of one patient, adenovirus type 3 from throat and rectal swabs of another patient, and adenovirus type 5 from the throat of a third patient (Table 3 ). The only significant finding in serological tests was a rise in neutralizing antibody to Coxsackie B5 from $<1: 10$ to $1: 30$ in one patient. There was a high incidence of anti-complementary activity in the complement-fixation antibody tests. These sera were not lipaemic or contaminated. Of fourteen acute-phase sera, twelve $(83 \%)$ were anti-complementary (titres $1: 2$ to $>1: 128$ ) and of sixteen convalescent-phase sera, seven $(43 \%)$ showed a similar abnormality. 
TABLE 3. Results of cultures for virus isolation. (From Dillon et al., 1974, by permission of the editor of the British Medical Journal.)

\begin{tabular}{|c|c|c|c|}
\hline \multirow[b]{2}{*}{ Culture system } & \multicolumn{3}{|c|}{$\begin{array}{l}\text { No. of isolates/ } \\
\text { No. of specimens }\end{array}$} \\
\hline & $\begin{array}{l}\text { Throat } \\
\text { swab }\end{array}$ & $\begin{array}{l}\text { Rectal } \\
\text { swab }\end{array}$ & CSF \\
\hline \multicolumn{4}{|l|}{ Cell cultures: } \\
\hline Primary monkey kidney (Patas) & $1 * / 16$ & $0 / 16$ & $0 / 1$ \\
\hline Primary monkey kidney (Rhesus)† & $0 / 6$ & & \\
\hline Continuous monkey kidney (V3A) & $0 / 39$ & $0 / 38$ & $0 / 1$ \\
\hline Continuous monkey kidney (Vero)† & $0 / 6$ & & \\
\hline Continuous rabbit kidney (RK13) & $0 / 39$ & $0 / 38$ & $0 / 1$ \\
\hline Human embryo lung (HEL/GOS) & $2 \ddagger / 29$ & $1 \S / 38$ & $0 / 1$ \\
\hline Human embryo lung (W138) $†$ & $0 / 6$ & & \\
\hline Human embryo kidney† & & $0 / 6$ & \\
\hline \multicolumn{4}{|l|}{ Organ cultures: } \\
\hline Human fetal trachea $\dagger$ & $0 / 6$ & & \\
\hline \multicolumn{4}{|l|}{ Animal inoculation: } \\
\hline Suckling mice & $1 * / 14 \pi$ & $0 / 149$ & $0 / 1$ \\
\hline
\end{tabular}

\section{Electron and immune electron microscopy}

Ill defined aggregates were found in some of the acute sera, particularly those shown to have anticomplementary activity.

\section{Other investigations}

Serum immunoglobulins were normal; circulating interferon was not found in acute-phase sera from patients nor in the supernatants of the lymphocyte cultures; there was no impairment of response to PHA stimulation of peripheral lymphocytes and no difference in unstimulated activity between patients and controls. In cultures of lymphocytes from three patients, there was a fall in cell numbers at first to about $20-25 \%$ of the original inoculum and then the remaining cells remained viable. In cultures from one of the patients, multiplication of cells occurred allowing subcultures to be made. Unfortunately, after 9,15 , and 18 weeks, these cultures were lost because of technical failure of the incubator.

\section{Affected children 1976-1978}

Although no children were affected during the 1970 outbreak of ENM at the Hospital for Sick Children, several children have recently been referred to the hospital with symptoms in keeping with this disease. Six children have been seen at the hospital, and full clinical details were available on a seventh child who was in the care of another unit. There were five boys and two girls. Average age at onset of symptoms was 11.7 years (range 9.3-13.0 years). In four cases, symptoms commenced in the
Autumn (September-October) of the year that the illness developed (two in 1976, and two in 1977) In two children the illness commenced in Januar $\overrightarrow{0}$ (1976 and 1977 respectively) and in the remaining. child, symptoms had developed in 1975. The two children (both boys) whose illness started in September, 1976, were cousins who had been on holiday together during August of that year and who subsequently returned to their homes, one in Hemel Hempstead, Hertfordshire, the other in Broadstairs, Kent. They both developed an identical illness at the same time in the September of the same year. Another child developed symptoms 1-2 weeks after his mother had started an identical illness and both were symptomatic when the child was referred for evaluation.

The symptoms and signs found in these children were very similar to those recorded in the adult patients seen at this Hospital in 1970. The main symptoms were headache, back and limb pains, lethargy and rapid fatigability on exercise. In addition, some complained of sore throats, nausea and abdominal pains. On examination, there was little to find except slight pharyngeal injection, moderate cervical lymphadenopathy and in some, slight pyrexia. These patients were also investigated but it is important to emphasize that when seen they were all past the initial acute phase of their illnesses. The illness in these children has run a protracted course and at least four continue to have symptomatic relapses up to two years or more after onset. The results of these investigations showed 
that two children had atypical lymphocytes in their peripheral blood and one a raised ESR. Liver function tests and serum CPK levels were normal. There were no consistent bacteriological findings. Virological investigations were non-contributory. One child had raised EB virus antibodies but of the six tested, none had any anti-complementary activity in serum and no lymphocytes would proliferate in vitro. Electroencephalography was undertaken on three of these children and in one the record was normal, in one compatible with ENM and in one, slightly abnormal but not typical of ENM.

\section{Discussion}

It was felt that the outbreak of this illness at the Hospital for Sick Children in 1970 was compatible with what is currently known as ENM (Parish, 1974). The protean symptomatology and contrastingly minimal clinical findings were characteristic. It was interesting that the ten or eleven commonest symptoms in this outbreak were almost identical to those reported in the Royal Free Hospital epidemic in 1955 (Medical Staff of the Royal Free Hospital, 1957). The physical findings were also in keeping with those reported in the literature but objective neurological abnormalities were less prominent than in some accounts (Pellew, 1951). The question of a functional disorder was considered early on in the 'epidemic' in the light of the suggestions by McEvedy and Beard (1970), in their retrospective investigation of the Royal Free Hospital epidemic, that epidemic hysteria was a likely explanation for that and other similar outbreaks of the disease. Although it was not considered that the illness had an hysterical basis, a deliberate decision was taken to limit the number of investigations undertaken, in order to prevent anxiety and fear in a theoretically susceptible population even though this would handicap attempts to discover the aetiology of the illness.

In spite of the essentially negative investigative results and the inability to isolate an infective agent, there was certain evidence which pointed to an infective aetiology. This included the undoubted physical signs in many patients, the biphasic pattern of the disease, the anti-complementary activity in sera, the presence of ill defined aggregates in some acute sera on immune electron microscopy, and the ability of some patients' lymphocytes to proliferate in vitro. The anti-complementary activity plus the immune electron microscopy findings suggest the presence of circulating immune complexes in the serum of these patients (Shulman and Barker, 1969). In addition, the findings of lymphocyte culture were interesting in view of the relative ease with which lymphocytes from patients with some lympho- proliferative disorders and infectious mononucleosis proliferate in vitro. The usefulness of this investigation was limited by the disappointing loss of the cultures due to a technical mishap at a relatively early stage. However, it was felt that these techniques might be the basis of further attempts to define the cause of this disease in future outbreaks.

The finding of sporadic cases of what appears to be this disease amongst children emphasizes that the condition is not limited to young adults, but can and does affect children as well (Sigurdsson et al., 1950; Wallis, 1957). It also emphasizes that ENM may be more common in the community than is realized but not so readily recognized because departures from the common norm are not so easily detected in that setting. Finally, medical personnel should be alerted to the possibility of this disease in both adults and children who present with the type of symptomatology described who sadly may have already been labelled as psychiatrically disturbed.

\section{Acknowledgments}

I should like to thank Dr Marguerite Pereira of the Central Public Health Laboratory, Colindale, London, for assistance with virological studies, Dr June Almeida of the Royal Postgraduate Medical School, Hammersmith Hospital, London, for immune electron microscopy studies, Dr R. N. P. Sutton of King's College Hospital, London, for EB virus antibody measurements, Mr G. T. Hawkins, FIMLT, for valuable technical assistance in laboratory investigations, and my colleagues, Professor J. A. Dudgeon and Dr W. C. Marshall at the Hospital for Sick Children, London, and Professor A. J. Steigman, Mount Sinai School of Medicine, New York, for their valuable support.

I should like to thank the British Medical Journal for permission to quote freely from the paper published by myself and others in that Journal (Dillon et al., 1974) and for permission to reproduce illustrations from that paper.

\section{References}

ACHeson, E.D. (1959) The clinical syndrome variously called benign myalgic encephalomyelitis, Iceland disease, and epidemic neuromyasthenia. American Journal of Medicine, 26, 569.

Almeida, J.D. \& Waterson, A.P. (1969) The morphology of virus antibody interaction. Advances in Virus Research, 15, 307.

Dillon, M.J., Marshall, W.C., Dudgeon, J.A. \& Steigman, A.J. (1974) Epidemic neuromyasthenia: outbreak among nurses at a children's hospital. British Medical Journal, 1, 301.

Henderson, D.A. \& Shelokov, A. (1959) Epidemic neuromyasthenia - clinical syndrome. New England Journal of Medicine, 260, 757, 814.

MCEVEDY, C.P. \& BEARD, A.W. (1970) Royal Free epidemic of 1955: a reconsideration. British Medical Journal, 1, 7.

Medical Staff of the Royal Free Hospital (1957) An outbreak of encephalomyelitis in the Royal Free Hospital Group, London, 1955. British Medical Journal, 2, 895.

PARISH, J.G. (1974) Epidemic neuromyasthenia: a reappraisal. Journal of International Research Communications, 2, 22.

Pellew, R.A.A. (1951) Clinical description of a disease resembling poliomyelitis seen in Adelaide, 1949-1951. Medical Journal of Australia, 1, 944. 
Shelokov, A. (1972) Epidemic neuromyasthenia (benign myalgic encephalomyelitis; Iceland disease). In: Infectious Diseases (Ed. by Hoeprich, P.D.), p. 1297. Harper \& Rowe, Maryland.

Shulman, N.R. \& BARKer, L.F. (1969) Virus-like antigen, antibody and antigen-antibody complexes in hepatitis measured by complement-fixation. Science. New York, etc., $165,304$.

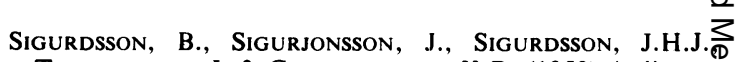
Thorkelsson, J. \& Gudmundsson, K.R. (1950) A disease epidemic in Iceland simulating poliomyelitis. American. Journal of Hygiene, 52, 222.

WALlis, A.L. (1957) An investigation into an unusuale disease seen in epidemic and sporadic form in generat practice in Cumberland in 1955 and subsequent years M.D. thesis, University of Edinburgh.

\section{Discussion}

Dr H. G. EASton (Glasgow): Why is it so unlike poliomyelitis where you get hundreds of cases in the community and perhaps one or two nurses? In this the ratio is, broadly speaking, the other way round. How does one explain that?

Secondly, why should none of the patients of these nurses in the hospital have got the disease? That is a puzzle, too, is it not?

Chairman: I referred to one point in my description of the outbreak in North Finchley between 1964 and 1967. It was noticeable that no sedentary persons were affected in that outbreak which included about 270 cases altogether. That might explain, too, the fact that no patients were affected in the Royal Free outbreak within the hospital. Is that correct, Dr Compston?

DR N. COMPSTON: There were very few, but of course the age group of the patients was very different from that of the medical staff, particularly the nursing staff. If this is a disease which is characteristic of the third decade of life it is well known that most patients in teaching hospitals are not in that age group.

Chairman: Another interesting little observation that I might make relates to a curious outbreak that occurred in Newcastle in 1959 in a teachers' training college. There were 120 student teachers in training at that college and $40 \%$ of them got this disease. The building was shared by a community of nuns who did the cooking for the college but only one nun was affected-again a relatively sedentary occupation.

DR D. PerRins (Oxford): You may remember that, in North Africa in the war, the incidence of infective hepatitis amongst officers was extremely high and amongst the men it was relatively low. That was due to the fact that there was communal washing-up in the officers' mess whereas the men washed up their own stuff. Possible that might apply in this situation.

DR G. PARISH: With regard to the hospital outbreak at Frohburg which occurred in the separate women's section, one would expect at least some of the women to be in labour (and consequently more physically active than other hospital patients).

DR M. J. Dillon: Apropos the question of, in our case children, not being affected at Great Ormond Street, we had at the time of this epidemic approximately 350 in-patients that we were looking at rather carefully during the whole course of the illness. As I mentioned earlier, they were not affected whereas we have been seeing children affected now. Wallis (1957) reported quite a high incidence of childhood involvement in Dalston, as also occurred in the Iceland epidemic (Sigurdsson $e t$ al., 1950). So I do not think that children are really excluded from the age range; I suspect they are just not picked upbecause they are not so often institutionalized in the way that many of the young adults are when these illnesses are being seen.

It might be that children in hospital are really no particularly active. They are lying down in bed, they arej clearly living a somewhat abnormal life, and it may be that muscular or physical activity plays some part in the susceptibility to infection. This may also be a reason why the Royal Free Hospital patients were not seemingly as affected as the staff. I have no hard data on this; it is 20 suggestion.

Chairman: I would just make the remark that un $-\overrightarrow{-}$ doubtedly physical activity plays a part. We notice this ino people who relapse. The relapses are practically alwayso precipitated by periods of excessive physical activity. One of our patients from 1955, who was then a medital student, has been a very active worker in this field a d was most eager to be present today. However, she

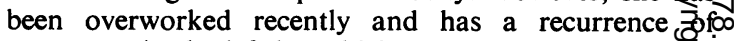
symptoms in the left leg which prevent her from drivitigo and she just cannot be present. This is undoubtedly due to a period of excessive activity.

As to the other point that was raised, you are no suggesting, are you, that this disease could be in any wayo an aberrant form of poliomyelitis?

DR EASTON: No, I was just comparing the one virus disease (if that is what it is) which seems to behave in such a totally opposite way from other virus diseases.

Chairman: There must be some curious relationshipo with poliomyelitis, as indicated by Dr Parish in his remark about the outbreak in Iceland in 1950. When? poliomyelitis was prevalent in Iceland in 1955 none of: the people who had had 'epidemic neuromyasthenia' in 1950 contracted that disease. We can no longer investi gate that very intriguing possibility of the connexiono with poliomyelitis virus for the simple reason that there are no more epidemics of poliomyelitis on which too base such an investigation.

DR EASTON: They behave in such totally oppositeo ways.

Chairman: Yes, it is very curious.

\section{References}

SigurdsSON, B., SigurJonsSON, J., SigurdSSON, J.H.J. స్ట ThORKELSSON, J. \& Gudmundsson, K.R. (1950) AO disease epidemic in Iceland simulating poliomyelitis American Journal of Hygiene, 52, 222.

WALLIS, A.L. (1957) An investigation into an unusual ${ }_{\mathscr{D}}^{\mathbb{D}}$ disease seen in epidemic and sporadic form in general practice in Cumberland in 1955 and subsequent years. M.D. thesis, University of Edinburgh. 